\title{
Determinantes sociales de la salud de la OMS en mujeres mexicanas con el virus de papiloma
}

\author{
Soltero-Rivera Silvia Guadalupe ${ }^{1, a}$, Cerda-Flores Ricardo Martin ${ }^{1, b}$, \\ Cárdenas-Villarreal Velia Margarita ${ }^{1, \mathrm{c}}$, Guevara-Valtier Milton Carlos ${ }^{1, \mathrm{~d}}$, \\ Paz Morales María de los Angeles ${ }^{1, e}$, Patton-Leal Adrián Carlos ${ }^{2, f}$, Ramírez-García Esther Justina ${ }^{3, g}$
}

\section{RESUMEN}

Uno de los temas centrales de la OMS es el análisis de los problemas de salud mediante el modelo de Determinantes Sociales de la Salud (DSS: inadecuadas condiciones económicas, ambientales y de alimentación). El sistema sanitario de la OMS ha asociado algunas enfermedades tal como el Virus de Papiloma Humano (VPH) con los DSS. Desde el punto de vista de investigación cuantitativa, los DSS reportados por la OMS son: 1) edad de inicio de la actividad sexual, 2) múltiples compañeros sexuales, 3) estrato socioeconómico y 4) sistema inmunitario debilitado. En México, hasta el momento las investigaciones cualitativas en población de mujeres mexicanas con VPH son nulas. Objetivos: identificar los DSS en mujeres mexicanas con VPH mediante una investigación cualitativa. Material y Métodos: diseño cualitativo-descriptivo. La población estuvo conformada por 1800 mujeres con VPH atendidas en consulta externa de un hospital de tercer nivel. De este total solo a 9 mujeres se seleccionaron para entrevista a profundidad (Método de Saturación) Minayo. Mediante el método de saturación se obtuvieron las unidades de significado, categorías y subcategorías. Resultados: se identificaron cinco categorías. 1. Determinantes conductuales, 2. Ingreso y situación económica, 3. Educación para la salud, 4. Estilos de vida y 5. Servicios de salud; utilizando el método de saturación. De estas categorías se obtuvieron nueve subcategorías. Las categorías con mayor saturación fueron: determinantes conductuales, (subcategorías: relación de pareja y relación sexual) e ingreso y situación económica (subcategorías: apoyo económico de pareja e ingreso personal).

PALABRAS CLAVE: Determinantes sociales de salud, virus del papiloma humano.

\section{Social determinants of health WHO Mexican women with papillomavirus}

\section{SUMMARY}

One of the central the mês of the WHO is the analysis of health problems through the Social Determinants of Health (SDH). The WHO health system associated with the DSS (inadequate economic, environmental and food) with some diseases. For the Human Papilloma Virus (HPV), the SDH reported by the WHO from the point of view of quantitative research are: 1) age of onset of sexual activity, 2) multiple sexual partners, 3) socioeconomic status and 4) system immune weaken ed. In Mexico, until this study itisnull qualitative research in Mexican women with HPV. Objectives: Identify the DSS in Mexican women with HPV through a qualitative research. Material and Methods: a descriptive qualitative design was carried out. The population consisted of 1800 women with HPV treated in an

\footnotetext{
Universidad Autónoma de Nuevo León. Facultad de Enfermería. Monterrey, México.

Secretaria de Salud. Hospital Regional Materno Infantil de Alta Especialidad. Guadalupe, México.

Universidad Nacional de Trujillo, Trujillo, Perú.

Maestría en Ciencias de Enfermería; ${ }^{\mathrm{b}}$ Doctorado en Ciencias Biológicas; ${ }^{\mathrm{c}}$ Doctorado en Enfermería ; ${ }^{\mathrm{d}}$ Doctorado en Educación

Doctorado en Educación; ${ }^{\mathrm{f}}$ Doctorado en Planificación y Gestión.
} 
outpatient tertiary hospital. Of this total only 9 women were selected for in-depth interview using the Minayo saturation method. Method of saturation, units of meaning, categories, and subcategories were obtained. Results: five categories of SDH (1. Behavioral determinants, 2.Income and economic situation, 3.Health education, 4.Lifestyles, and 5. Health services) were identified. Nine subcategories were obtained by the saturation method. The categories with higher saturation were: behavioral determinants (subcategories: relationship and sex) and income and economic situation (subcategories: financial support and personal income couples).

KEYWORDS: Social determinants of health, human papillomavirus.

\section{INTRODUCCIÓN}

Los determinantes sociales de salud (DSS) son tema central de la Organización Mundial de la Salud (1). Es evidente que aún se trata de un campo de estudio en construcción, la relación entre las condiciones de vida de las personas y su estado de salud se estableció desde las primeras décadas del siglo XIX, el sistema sanitario puede considerarse como un determinante social de la salud, en el mismo siglo se evidenció que las enfermedades en general estaban asociadas con las inadecuadas condiciones económicas, ambientales y de alimentación, aumentando la tasa de mortalidad, estas condiciones aunadas a los factores psicosociales contribuyen ya sea de manera directa o indirectamente en la prevención y tratamiento del virus del papiloma humano (VPH) (2).

Entre las mujeres en edad fértil, el cáncer en el cuello del útero tiene una tasa de mortalidad observada de 41.88 por cada 100 mil mujeres (3). En México se considera un problema de Salud Publica debido a la magnitud en el número de casos de mujeres con VPH, alrededor de $11 \%$ de mujeres con vida sexual activa tienen el VPH. Es una de las infecciones de transmisión sexual (ITS) más frecuentes en la mujer (4). Misma que puede ser asintomática durante al menos 6 semanas a 6 meses después de resultar infectado, tal vez no se evidencien durante años (5).

Se piensa que los DSS como la edad del inicio de actividad sexual, múltiples compañeros sexuales, estado socio económico, un sistema inmunitario debilitado debido a una enfermedad o medicamento, consumo de alcohol y tabaco están involucrados directamente en el desarrollo de lesiones pre invasoras o invasoras de cérvix, como la lesión de VPH, independientemente de otros factores. Las ITS se presentan en mujeres de 10-55 años y en el $90 \%$ de los casos algún tipo de displasia cervical.

Datos del Instituto Nacional de Estadística y Geografía sobre tumores malignos menciona otro tipo de cáncer que afecta a la población femenina, el cáncer cervicouterino que se origina generalmente por la exposición del VPH del que existen más de 150 variaciones, 15 son responsables de la mayoría de los casos de cáncer cervicouterino, vagina, pene, vulva, ano y orofaríngeo (6).

Según el Centers for Disease Control and Prevention, el VPH puede causar aislamiento social, disminución de la función sexual y otros problemas psicológicos que permanecen ocultos, lo cual desencadena modificaciones en la forma en que la mujer percibe su sexualidad, generando sentimientos de culpabilidad o rechazo hacia la pareja.

Breilh en el año 2003 en sus trabajos sobre la necesidad de superar la visión de la salud como un tema de enfermedad, hace referencia hacia la apertura de los DSS, por lo que propone ver la salud desde su complejidad no solo de la vida individual, sino también abarca lo que suceda en las clases sociales, en los grupos socioeconómico en el que uno forma parte y a la vez ese grupo socioeconómico forma parte de la sociedad, el considera tres dimensiones: La dimensión general que cuenta con tres elementos (económica, política y cultural), la dimensión particular de los grupos y la dimensión individual de las familias. Dado lo anterior, el objetivo del presente estudio fue identificar los DSS en mujeres con VPH de un hospital público del Noreste de México (7).

\section{Antecedentes de la investigación y justificación del problema}

De acuerdo con el concepto de "Determinantes sociales de salud", en los que se destacan en la actualidad, los factores socioeconómicos, entorno social, apoyo social y el entorno relacionado con la salud se observa la magnitud de las limitaciones de las intervenciones de salud orientadas a disminuir los riesgos individuales de enfermar y morir (8).

La atención médica no es el principal condicionante de las personas, sino que aquella está determinada en gran parte por las condiciones sociales en las cuales se vive y 
se trabaja, estos factores permiten que las personas permanezcan sanas y la atención medica ayuda cuando se enferman. En México, las mujeres de entre 15 y 45 años de edad se encuentran en riesgo latente al $\mathrm{VPH}$, alrededor de $11 \%$ de mujeres con vida sexual activa tienen el VPH, es una de las ITS más frecuentes en la mujer. Las que puede ser asintomática durante al menos 6 semanas a 6 meses después de resultar infectado, tal vez no se evidencien durante años (5).

Estudios epidemiológicos indican que uno de los factores de mayor riesgo en el desarrollo de lesiones pre invasoras o invasoras de cérvix, es la lesión de VPH, independientemente de otros factores de riesgo tales como: edad de inicio de actividad sexual, múltiples compañeros sexuales, estado socio económico si tiene un sistema inmunitario debilitado debido a una enfermedad o medicamento, consumo de alcohol y tabaco. Asimismo esta ITS se presenta en mujeres 10-55 años y en el 90 por ciento de los casos se presenta algún tipo de displasia cervical (3).

El virus del papiloma humano (VPH) produce una compleja infección de transmisión sexual (ITS) que ocasiona cáncer cérvico-uterino en la población mexicana. Datos estadísticos del Instituto Nacional de Estadística y Geografía reportan que del $100 \%$ de la población de los Estados Unidos, de 2004 al 2008, estimó que cada año ocurren cerca de 33,300 casos de cáncer asociados al VPH(3). Alrededor de 21300 de estos casos corresponden cada año a mujeres y 12100 cada año a hombres. Por lo tanto, la infección por VPH se presenta cada vez más en la población a partir de los 15 años de edad.

Para el año 2001 se realizó un estudio en Canadá en el que se determinó que en el grupo etario comprendido los $0 \mathrm{y}$ los 10 años de edad del $86 \%$ de la muestra eran positivas al VPH, en relación con otros grupos de edad y las firmas clínicas que predominaban eran las verrugas y papilomas laríngeo. De los virus aislados el 68\% eran de origen genital, observándose con más frecuencia el tipo 6 y 11(9). Datos del Instituto Nacional de Estadística y Geografia sobre tumores malignos hace referencia a otro tipo de cáncer que afecta a la población femenina es el cervico-uterino, que se origina generalmente por la exposición del Virus del Papiloma Humano (VPH) del que existen más de 150 variaciones, cuya característica principal es la producción de verrugas genitales, solo 15 son responsables de la mayoría de los casos de cáncer cervico-uterino, vagina, pene, vulva, ano y orofaríngeo $(3,6,10)$.

El personal de enfermería se encuentra en un período cru- cial en el desarrollo del conocimiento enfermero, puede afirmarse con total certeza que la ciencia de enfermería se halla inmersa en plena revolución científica, los cambios producidos en el contexto de la salud como la transición de la época en donde las enfermedades infectocontagiosas ocupaban un lugar importante y a la época donde fueron sustituidas por enfermedades crónicas trayendo un cambio considerable en el abordaje de la salud y por supuesto un replanteamiento para la disciplina de enfermería sobre un nuevo contexto (10).

Dentro del área de salud es imprescindible remarcar a enfermería como disciplina científica integrada por ciencia y profesión en el que se pretende obtener como aporte a la disciplina conocimientos que se apliquen a partir de una forma particular de ver una relación sujeto-objeto que permitan desarrollar su praxis, teniendo fundamentos que la respalden, enfermería entrelaza presente, pasado y futuro (11).

Se pretende que los resultados puedan arrojar datos para que permitan implementar propuestas de prevención y promoción, con enfoques que consideren no solamente el aspecto biológico, sino la complejidad del fenómeno,ya que las infecciones de transmisión sexual constituyen un problema de relevancia para la comunidad y esto apoyará para fomentar medidas preventivas por medio de información, cuidado y educación. La información es un derecho universal que tienen los pacientes y esta debe ser confiable para favorecer un desarrollo integral siendo capaz de producir cambios educativos en los estilos de vida de la población beneficiaria.

En las sociedades donde la salud se considera principalmente un asunto privado, el tema de la responsabilidad personal, se ha desplegado políticamente a fin de absolver al gobierno de la responsabilidad de abordar las desigualdades de salud y de responder de manera más activa a las necesidades de salud de grupos pobres, vulnerables y excluidos, por lo que existe una relación estrecha entre los determinantes sociales y la equidad en salud (12).

Es de gran utilidad, brindar el conocimiento obtenido para aportar estrategias y a partir de ellas desarrollar proyectos que contribuyan para aportar solución a los problemas detectados y mejorar la comprensión de los fenómenos que se relacionan a través de recabar información valida fijando la mirada a optimizar el proceso de ésta investigación. La revolución de la información contribuye en el desarrollo de conocimientos para la disciplina de ciencias de enfermería, por lo que no puede existir actualización sin 
una debida información. Se pretendió obtener información para difundir, promocionar y generar prevención.

Es de importancia trascendental que las poblaciones y comunidades reciban la información de forma debida y específicamente sobre el virus del papiloma humano en vista de que el número de casos de mujeres infectadas con el VPH se ha mantenido elevado a través de los últimos años, nos surge la inquietud para estudiar los determinantes sociales que han mantenido éstos niveles altos, es necesario conocer toda la evolución compleja de los determinantes que dependen de las condiciones que favorecen su expresión.

\section{Relevancia del estudio}

Para la Salud Pública es de gran importancia considerar los cuidados de enfermería para el control del incremento considerable en la tasa de VPH, el conocer los determinantes sociales que predominan en la población mexicana permitirá que la enfermera brinde cuidado integral y holístico en los aspectos: bio-psico-social por medio de proporcionar información de los cuidados y factores de riesgo existentes para prevenir la enfermedad, insistir en los cuidados adecuados preventivos para el hogar, sobre higiene, alimentación, actividad sexual, evitar hábitos re riesgo como múltiples parejas sexuales y adicciones entre otros, siendo estos factores de riesgo básicos que se encuentran inmersos dentro de los determinantes conductuales, de los ingresos y situación económica, estilos de vida y servicios de salud, es de considerable importancia para mejorar la economía del país por que disminuyen los casos atendidos en un tercer nivel de atención de casos avanzados y hospitalizaciones por causa de algún tipo de VPH que condicione cáncer.

En el Hospital Regional Materno Infantil de Alta Especialidad en Guadalupe Nuevo León en el año 2014 se obtuvieron 684 pacientes que se detectaron por primera vez con VPH positivo, 180 como subsecuentes de VPH, 6 pacientes de NIC 1 detectados por primera vez y 747 subsecuentes, 1 detección por primera vez de NIC II y 128 subsecuentes, 1 detección de NIC III y 53 subsecuentes, todas con Virus de Papiloma humano positivo, dando un total de 1,800 pacientes atendidas por VPH, por lo que siendo datos elevados de pacientes diagnosticadas con $\mathrm{VPH}$.

La real importancia se encuentra en la prevención desde un primer nivel de atención, por lo que se propone abarque de lo general a lo particular, de un macro sistema al micro para crear un impacto mayor en la población, mejorar la equidad en salud por medio de un mayor enfoque a la población marginada que es la de mayor vulnerabilidad, manteniendo un sistema de salud real y gratuito cerca de su lugar de residencia con seguimiento a su diagnóstico.

El objetivo del estudio fue encontrar los determinantes sociales que condicionan infecciones recurrentes de papiloma humano en mujeres mexicanas.

\section{Objetivos especificos}

- Identificar los determinantes sociales que condicionan infección por virus del papiloma humano en mujeres Mexicanas.

- Analizar los determinantes sociales que condicionan infección por virus del papiloma humano en mujeres Mexicanas.

\section{MATERIAL Y MÉTODOS}

\section{Tipo de estudio}

La presente investigación es un estudio cualitativo de tipo descriptivo- estudio de caso, que permitirá explicar e identificar los determinantes sociales en mujeres Mexicanas y cómo éstos contribuyen para presentar una reinfección por el Virus del Papiloma Humano, además permitirá conocer el fenómeno en estudio en su contexto natural (12).

\section{Método, técnica y dinámica.}

El método que se utilizó fue el método descriptivo a través del estudio de casos ya que según Yin en los estudios de caso es posible hacer observación directa sobre los fenómenos para generar conocimiento sobre características significativas de eventos vivenciados (12). Además, en el método de estudio de caso los datos pueden ser obtenidos desde una variedad de fuentes, tanto cualitativas como cuantitativas; esto es, documentos, registros de archivos, entrevistas directas, observación directa, observación de los participantes e instalaciones u objetos físicos (13).

\section{Escenario de Investigación}

La investigación se llevó a cabo en el Hospital Regional Materno Infantil de Alta Especialidad, orientado hacia la atención de mujer y niño, enfocado al diagnóstico y tratamiento del virus del papiloma humano, enfermedades de transmisión sexual, detección de mujer con VIH y embarazo de alto riesgo, para no interferir con los proce- 
dimientos se solicitó el consultorio anexo al departamento de Colposcopias de dicho hospital, el consultorio cuenta con computadora silla, descanset, cama de exploración, lámpara de chicote y escritorio, se leyeron y firmaron las hojas de consentimiento informado, y se mantuvo en todo momento en cuenta los derechos del paciente y su privacidad, se solicitó grabar la conversación a la participante para posteriormente transcribir.Además se contó con una guía básica de recolección de datos. Los sujetos de investigación fueron nueve mujeres con diagnóstico positivo de VPH, que asistieron al Hospital Regional Materno Infantil de Alta Especialidad en el municipio de Guadalupe, Nuevo León, México.

La selección de las nueve mujeres participantes se realizó a través de un muestreo intencional no aleatorio, dinámico y secuencial. Para determinar el tamaño de la muestra, se tomó en cuenta el principio de saturación de datos (12).

Se realizaron en todas ellas entrevistas semiestructuradas las cuales fueron grabadas (Modelo Sony ICD-PX312F) durante un periodo de un mes para posteriormente transcribirlas.

La investigación se llevó a cabo en un hospital de tercer nivel orientado hacia la atención de mujer y niño, enfocado al diagnóstico y tratamiento del virus del papiloma humano, enfermedades de transmisión sexual y detección de mujer con VIH para no interferir con los procedimientos se solicitó el consultorio anexo para tal fin al departamento de colposcopia de dicho hospital. Se leyeron y firmaron las hojas de consentimiento informado, y se mantuvo en todo momento en cuenta los derechos del paciente y su privacidad, se solicitó grabar la conversación a la participante.

La pregunta orientadora fue ¿Qué determinantes sociales condicionan infección por Virus de papiloma humano? Posteriormente, esta pregunta se fraccionó en cuatro preguntas para facilitar la comprensión de la participante. A continuación, se describen las cuatro preguntas ¿Qué conoce usted sobre el VPH? ¿Qué cree usted que produce el VPH? ¿Por qué cree que a enfermando usted del VPH? ¿Cómo es la relación de pareja con su esposo después que supo que usted tenía VPH?

\section{Rigor y consideraciones éticas}

Consideraciones éticas: se tomará un código de conducta formal de acuerdo al momento de la entrevista y previa autorización por el comité de ética de la Universidad de Trujillo; Perú y del Hospital Regional Materno Infantil de Alta Especialidad en donde se llevará a cabo la entrevista, tomando en cuenta los valores y manteniendo una postura ética en todo momento. El presente estudio se realizó conforme a lo que dispone el Reglamento de la Ley General de Salud en Materia de Investigación de la Secretaria de Salud.

Se contó con el consentimiento informado y por escrito de los participantes del estudio, además del dictamen favorable de la Comisión de Investigación y Ética de la Universidad de Trujillo así como de la institución participante, después de tener la autorización de las autoridades correspondientes se brindó una información clara y completa del estudio a los participantes del estudio.

Protegiendo la privacidad del participante dado que no se registró el nombre en ninguna parte del estudio, además se indica el lugar donde se desarrolló la entrevista y los datos se manejaron en forma confidencial. Este tipo de estudio se considera sin riesgo dado que no se realizó ninguna intervención, sólo se aplicó un cuestionario a través de una entrevista guiada. Se le aclaró que podrá retirarse del estudio en el momento que lo desee, además se le explico el objetivo del estudio.

\section{Consideraciones éticas}

Consentimiento informado: en la cual se documenta la decisión de participar de manera voluntaria después de haber recibido una explicación sobre la investigación para grabar sin afectar la credibilidad (valor de la verdad, cuando las personas reconocen que lo leído es cierto).

Dignidad humana: derecho a la autodeterminación, consistirá en dejar que el participante exprese lo que siente, lo que piensa y lo que hace sin ningún tipo de coacción, así mismo se asegurará un ambiente tranquilo, cómodo y lejos de bullicio de la gente.

Principio de Justicia: este principio incluye el derecho de sujeto a un trato justo y la privacidad, por lo cual el participante, en todo momento será considerado, en la investigación ante todo como ser humano, este principio abarca las siguientes : consideraciones de rigor científico Credibilidad: está dada por la verdad establecida mutuamente entre el investigador y el informante, es la verdad conocida, sentida y experimentada por la gente que está siendo estudiada.

Transferibilidad: posibilidad de transferir el estudio cualitativo hacia otros contextos, ámbito o grupos; desde que se obedece la preservación de los significados, inter- 
pretaciones e interferencias particulares. Se necesita que se describa densamente el lugar y las características de las personas donde el fenómeno fue estudiado.

Auditabilidad: está referida a la comprensión de los diferentes momentos y de la lógica del trabajo de investigación por otros lectores e investigadores podría seguir la sucesión de eventos en el estudio y con el entendimiento de su lógica, se cumple en la medida que se describe detalladamente el abordaje teórico y metodológico, el análisis, la discusión y las condiciones finales del trabajo, con el fin de que otro investigador examine los datos y pueda continuar con investigaciones similares.

\section{RESULTADOS}

Se encontró que la edad actual de las participantes infectadas con VPH predomina entre 34 a 49 años con un $64 \%$, el estado civil casada con un $37,5 \%$, de unión libre con $35,5 \%$, y número de hijos de $3-4$ con $48 \%$. Se aprecia que el IVSA con mayor relevancia predomina en la adolescencia, de 14-16 años con un 40\% mientras que de 17 a 19 años encontramos el 31,5\%. Las parejas sexuales de la población varían de entre 1 pareja sexual, hasta 20 parejas sexuales, predominando entre 1-2 parejas sexuales con el $57,5 \%$ y de 3-4 parejas con el 30\%. El tipo de Displasia que se encontró fue el de NIC 1 más VPH positivo con mayor relevancia con un $81 \%$, en segundo lugar, el VPH sin complicaciones al momento con un $10 \%$.

Las Categorías (C) y Subcategorías (SC) que surgieron de las entrevistas a pacientes con VPH en el HRMIAE se clasificaron como se señala a continuación: $\mathrm{C} 1$ Determinantes conductuales - SC1.1Relación de pareja-SC1.2 Relación Sexual, C2Ingresos y situación económica - SC2.1 Apoyo Económico de Pareja - SC2.2Ingreso Personal, C3 Estilos de Vida - SC3.1 (Des) conocimiento para el cuidado SC3.2 (Des) conocimiento de la Enfermedad, C4 Servicios de salud-SC4.1 (des) Atención de los Servicios de Salud - SC4.2 Información de los Servicios de Salud.

Breilh, refiere que el paradigma lineal de las causas y factores de riesgo al asumir una concepción de la realidad $\mathrm{y}$ al sujeto situado por fuera y aparte de la realidad social y la naturaleza, es el caso de una práctica focalizada en dichos factores de acuerdo a la magnitud que los expresan con sus efectos, hace referencia a los efectos y causas al asumir factores de riesgo, situación que, de acuerdo a los cambios sociales, económicos, políticos y otros, tiene que considerarse en forma integral considerando los determinantes que emergen en cada situación (7).

La primera categoría del estudio considera los determinan- tes conductuales con las subcategorías relación de pareja y relación sexual.

En los determinantes conductuales, se define el campo de aplicación propio de la Psicología de la Salud, acerca del proceso Salud-Enfermedad, los cuales constituyen condiciones fundamentales que facilitan el surgimiento de la Psicología de la Salud. Se describen en los niveles de promoción de la Salud, y de prevención primaria, secundaria y terciaria de la enfermedad; se enfatiza el papel que tiene el comportamiento como factor determinante del curso que tome el proceso Salud-Enfermedad, de la responsabilidad que tiene el individuo para con su propio proceso vital.

Actualmente los determinantes de salud caracterizados por una deficiente relación de pareja, conflictos dentro de la misma relación y como consecuencia infección de transmisión sexual; determinante biológico, individual que según Breilh (7) no siempre se visualizan, sin embargo, la conjunción con otros determinantes establece su aparición. Situación que también se muestra en el estudio, según los discursos de las participantes:

“(...) soy respetuosa con él, le doy su lugar por ser padre de mis hijos, nunca sali con nadie mientras estuve con él, yo confiaba en mi esposo y él me infecto ya no estoy con mi esposo, estoy con otra persona. Estamos bien y él sabe de eso (silencio) él dice que él está conmigo y pues él me va a apoyar" (María).

“(...) yo ya me habia hecho el Papanicolaou cuando tuve a mi hija y yo habia salido bien y yo no mantuve ni una relación ni nada en ese tiempo, puede ser eso, él ya es mayor y trabaja en estados unidos, creo q él ha tenido allá otras parejas, pero él no dice nada" (...). Se acaba de ir la semana pasada, viene cada mes dura una semana aqui y se va," (Juanita).

Relación Sexual: las condiciones de la sexualidad desde el vínculo de pareja, así como la exposición a microclimas, acceso a una cuota familiar alimentaria, participación en el trabajo familiar no remunerado. En definitiva, en la unidad familiar se da por parte del proceso de transformación de las condiciones más generales de reproducción social, las condiciones materiales y sociales de existencia, es estrategias de supervivencia de los individuos en el pequeño grupo Breilh (7). Esta se ve de manera directa o indirectamente afectada con la desigualdad social y de género que existe en nuestro país.

“(...) no me cuide al tener relaciones sexuales con preservativo o algo, en segundo porque yo creía en mi pareja, creía que no tenía otras personas con relaciones sexuales 
anteriores a mi o que también se había cuidado" (Valeria). (...) me dijeron que el virus se contagia con una relación sexual, (...), me hicieron la cirugía, me quitaron ese pedacito y me cauterizaron, me dijeron que no tuviera relaciones sexuales por tres meses (María).

(...) yo no tuve ningún contacto sexual, por 2 años, estaba sola entonces reinicio lo sexual y de repente ya salgo contagiada (Juanita).

(...) Mi esposo no quería que viniera por resultados al hospital, porque no hemos tenido relaciones y dice: Después te van a decir que otros tres meses más y se enoja porque dice que ya llevamos mucho sin relaciones (Laura). (...) me siento incomoda al momento de las relaciones, para ir al baño, por las verrugas ya que me sale sangre al tocarlas o rascarlas (...) me las quitaron durante el embarazo 3 veces (Valeria).

Estos testimonios que se obtuvieron como resultado de las entrevistas reflejan que al verse la relación de pareja afectada surgen situaciones de infidelidad, teniendo falta de compromiso en su relación de pareja y sintiendo como no estable una relación de unión libre, el 30\% de los casos atendidos en el hospital regional Materno Infantil de Alta especialidad han tenido más de 3 a 4 parejas sexuales, inclusive de 7 a 20 parejas con un 4,5\%.

Breilh (7) en su investigación sobre la determinación social de la salud como herramienta de transformación hacia una nueva Salud Pública: En el paradigma de la determinación social se plantea en la interface del materialismo crítico, la economía política y la ecología política en su diálogo con una versión crítica de las ciencias de la salud, el ambiente y la sociedad. Se propone descifrar el movimiento de la vida, de su metabolismo histórico en la naturaleza, de los modos de vivir típicos (económicos, políticos, culturales) y del movimiento de los geno-fenotipos humanos, en el marco del movimiento de la materialidad social cuyo eje es la acumulación de capital, nombre que toma en nuestras sociedades la reproducción social; la que se ve reflejado en la actualidad dentro de la sociedad y de las mismas familias, en la economía, en el apoyo económico y sostén económico, en el que los ingresos son inferiores a los egresos percibidos.

“(...) él me deposita pero con los niños tenemos gastos de hecho aqui en este hospital nacieron mis niños, aqui llevaron su control, hay muchos gastos por qué pago renta aqui y me acaba de llegar el recibo de la luz de $\$ 3000$, y luego la renta \$2000 y luego mi hija está en un colegio, hoy tocó el pago de $\$ 1250$ pero ciertos meses se paga doble por las vacaciones y hoy toco doble mensualidad porque ya viene abril y es de vacaciones, y entonces pues pago transporte y los servicios de la casa, despensa, yo tengo una extensión de la tarjeta, el me deposita, y él tiene cierto límite que puedo estar sacando en la tarjeta, ósea que puedo sacar más y luego él lo repone y así esta y aparte pues en una urgencia o algo pues tiene que tener algo alli"'(Juanita).

Con suficiente evidencia, el dinero y los recursos, las condiciones de vida determinan la manera como las personas enferman y mueren. Cabe señalar que las dos vertientes de la epidemiología social reconocen la importancia del campo social de la salud en interacción con la biología, relevan la relación entre pobreza, condiciones de trabajo y enfermedad como elementos clave del proceso salud enfermedad y establecen que los procesos sociales macro condicionan los patrones de salud y enfermedad en que las condiciones de vida determinan la manera como las personas enferman y mueren y, a su vez, propone mejorar dichas condiciones, y luchar contra la distribución desigual del poder, para así eliminar las desigualdades injustas en salud, en una generación $(7,14)$.

Factor económico y distribución del dinero interno familiar se considera deficiente o nulo dado que algunas de estas parejas se encuentran con doble vida familiar y $\sin$ apoyo económico a una de las dos familias, mujeres separadas o divorciadas sin trabajo estable o que aun dependen económicamente de sus padres aunado los hijos que ellas tienen, mujeres viudas sin pensión alimenticia en las que se ve claramente la desigualdad social y económica.

La OMS promovió, desde su fundación en 1948, en diferentes conferencias y reuniones internacionales con el objetivo de marcar la orientación para la salud universal, el lema "Salud para todos en el año 2000", la Conferencia de Alma-Ata, la Conferencia de Ottawa y la Conferencia de Yakarta, son algunos de los eventos que desde la OMS promovieron las estrategias derivadas de esta nueva definición de la salud (1).

En la categoría de estilos de vida con la subcategoría de falta de conocimiento para el cuidado, en la que se hace notar el estrecho vínculo al que pertenece la educación con el cuidado que cada uno de ellos tiene para su salud. (...) me mandan a hacer el examen del SIDA (...) a uno se le vienen muchos pensamientos $q$ dices tú (...) tienes ya una bacteria, si por una relación no cuidada o por lo que haya sido... luego te mandan a hacerte estudios para detectar SIDA (mimi).

(...) no tuve las debidas precauciones, por confiar en la persona no indicada, porque de hecho yo cuando tenía relaciones, las tenía con preservativo y con el no, allí 
falle (...) alli fue donde me contagie. No entiendo, y me da coraje porque me enferme (María).

(...) no tenía mucho conocimiento en si porque pues nunca pensé tener esa enfermedad, de cómo cuídame estando embarazada y como cuidar a mi bebe para que no se contagiara (Valeria).

(...) yo me asusté cuando me dijeron que tenía eso y me dijo que era por transmisión sexual y que la mayoría de las veces es la pareja la que nos transmite eso, porque cuando me dijeron que tenía VPH, yo pensé que ya tenía cáncer (Laura).

La adolescencia es el periodo de la vida en el cual se adquiere la capacidad reproductiva y se hace la transición de la niñez a la adultez, abarca las edades entre 10 y 19 años y se caracteriza por la sucesión de importantes cambios fisiológicos, psicológicos y sociales en el ser humano. En esta etapa, las intervenciones en materia de salud sexual son vitales para el desarrollo físico, mental y social del adolescente y evitar o reducir consecuencias adversas de comportamientos sexuales no saludables como las infecciones de transmisión sexual (ITS)(14).

El IVSA con mayor relevancia según datos obtenidos, predomina en la adolescencia cuando la mayoría de las personas aún no mantienen un trabajo estable ni una escolaridad terminada, entre 14 a 16 años tenemos un $40 \%$ de mujeres que inician su vida sexual activa con VPH diagnosticado en las edades de 17 a 19 años el $31,5 \%$ que inicio su VSA.

En la categoría de los servicios de salud y las subcategorías (des) atención de los servicios de salud e información de los servicios de salud nos relacionamos con la OMS (1). En el que hace referencia que, en todo el mundo, las personas socialmente desfavorecidas tienen menos acceso a los recursos sanitarios básicos y al sistema de salud en su conjunto. Es así como enferman y mueren con mayor frecuencia que aquéllas que pertenecen a grupos que ocupan posiciones sociales más privilegiadas. Esto se hace más crítico en algunos de los grupos más vulnerables. Estas inequidades han aumentado a pesar de que nunca antes han existido en el mundo la riqueza, los conocimientos y la sensibilidad e interés por los temas que atañen a la salud como en la actualidad, en el presente estudio encontramos desde NIC I hasta NIC III.

De acuerdo a lo anterior, podemos concluir que la reincidencia de conductas sexuales de riesgo es muy común entre las mujeres que han sido diagnosticadas por VPH por lo que a la larga muchas de estas regresan a los servi- cios de salud con problemas más serios como displasias severas y cáncer cervical.

La posición socioeconómica tiene una influencia sobre la salud a través de determinantes más específicos o intermediarios, estos factores intermediarios son condiciones materiales, estilos de vida, como la situación laboral y la vivienda, circunstancias psicosociales y también factores conductuales como la exposición a factores de riesgo específicos, el abordar el gradiente socioeconómico constituye un modelo más integral para intervenir en las inequidades de salud.

Con el entorno social más amplio y a través de acciones sectoriales e intersectoriales, el soporte de los programas de seguridad social y el financiamiento equitativo de la atención son componentes fundamentales por lo que concuerdo con Marmot en la propuesta que para tener una repercusión real sobre las inequidades en salud, es necesario alcanzar la cobertura universal y abordar los DSS mediante la acción conjunta y coordinada, dichas medidas, exigen conocimientos profundos, históricos, de cambio social, para producir resultados a largo plazo, esto implica un compromiso de la sociedad en conjunto con las generaciones futuras (14).

\section{REFERENCIAS BIBLIOGRÁFRICAS}

1. Organización Mundial de la Salud. Social determinants of health the solid facts. BogotA, Colombia: OMS; 2003.

2. Turner B. The history of the changing concepts of health and illness outline of a general model of illness categories. En: Albrecht G, Fitzpatrick R, Scrimshaw S(Eds.), Handbook of social studies in health and medicine. London: SAGE Publications Ltd ;2000. p. 9-24 doi: http:// dx.doi.org/10.4135/9781848608412.n2

3. Hernández CG, Smith JS, Lorincz A, Arreola ECh, Lazcano E, Hernández MÁ, Salmerón J. Prevalencia de infección por vírus de papiloma humano (VPH) de alto riesgo y factores asociados en embarazadas derechohabientes del IMSS en el .estado de Morelos. Salud Publica. 2005; 47(6): 423-429.

4. Lorinez A. Molecular methods for detection of Human Papilomavirus infection. Obstet Gynecol clin North Am.1996; 23:3707-715.

5. Walboomers JM, Jacobs MV, Manos MM, et al. Human Papillomavirus is a necessary cause of invasive cervical cancer worldwide. J Pathol. 1999; 189(1):13-19.

6. Instituto Nacional de Estadística y Geografía. Estadísticas sobre tumores malignos. Lima, Perú: Instituto Nacional de Estadística y Geografía; 2009.

7. Breilh J. Una perspectiva emancipadora de la investigación e incidencia basada en la determinación social de salid. ALAMES. 2003; 155: 1-22. 
8. Tarlov A. Social determinants of health: the sociobiological translation. In: Blane D, Brunner E, Wilkinson R, eds. Health and social organization. London: Routledge; 1996.p. 71-93.

9. Feoli, Fonseca JC, Oligny L, Sijmard P, Falcon S, Yotov W. Human Papilomavirus (VPH) Study of 691 pathological specimens from Quebec by PCR-Direct sequencing approvach. J-Med. Virol. 2006; 63: 284-292.

10. Instituto Nacional de Estadística y Geografía. Estadísticas a propósito del día mundial contra el cáncer. Lima, Perú: Instituto Nacional de Estadística y Geografía; 2011.

11. Vílchez V, Sanhuesa A. Enfermería una disciplina Social. Enfermería en Costa Rica. 2012; 32(1):81-88.

12. Vega J, Solar O, Irwin A. Equidad y Determinantes sociales de la salud: conceptos básicos, mecanismos de producción y alternativas para la acción. Ginebra: Equipo de Equidad en salud de la ORGANIZACIÓN Mundial de la Salud; 2011.p. 9-18.
13. Martínez P (2006). El método del estudio de caso. Estrategia metodológica de la investigación científica. Pensamiento \& Gestión. 2006;20(1):165-193.

14. Marmot M, Pellegrini AF, Vega J, Solar O, Fortune K. Acción con respecto a los determinantes sociales de la salud en las Américas. Revista Panamericana Salud Publica. 2013; 34(6): 382-384.

\section{Correspondencia:}

Dra. Silvia G. Soltero-Rivera.

Email:silviasoltero1972@hotmail.com

Fecha de Recepción: 20 de noviembre del 2015

Fecha de aceptación: 10 de diciembre del 2015 